\title{
Alterstice
}

Revue internationale de la recherche interculturelle

International Journal of Intercultural Research

Revista International de la Investigacion Intercultural

\section{Sortir la violence de sa vie, sans sortir de l'autochtonie : l'importance de mieux comprendre les besoins des femmes autochtones en milieu urbain}

\section{Catherine Flynn, Geneviève Lessard, Lyse Montminy et Renée Brassard}

Volume 3, numéro 2, 2013

Violence conjugale et diversité culturelle

URI : https://id.erudit.org/iderudit/1077519ar

DOI : https://doi.org/10.7202/1077519ar

Aller au sommaire du numéro

Éditeur(s)

Alterstice

ISSN

1923-919X (numérique)

Découvrir la revue

Citer cet article

Flynn, C., Lessard, G., Montminy, L. \& Brassard, R. (2013). Sortir la violence de sa vie, sans sortir de l'autochtonie : l'importance de mieux comprendre les besoins des femmes autochtones en milieu urbain. Alterstice, 3(2), 37-50. https://doi.org/10.7202/1077519ar

\section{Résumé de l'article}

Cet article présente les résultats d'une étude s'inscrivant dans une action concertée portant sur la violence conjugale envers les femmes autochtones du Québec. Notre recherche a porté sur les besoins et l'expérience de ces femmes en milieu urbain. Le corpus était constitué de trois groupes de discussion comprenant 24 hommes et femmes autochtones de Québec et de Montréal, tous concernés par la problématique de la violence conjugale. L'analyse a permis de dégager trois besoins en matière d'aide psychosociale : faire connaître la situation et le vécu des femmes autochtones victimes de violence conjugale, bénéficier d'une aide adaptée à la réalité culturelle des Autochtones et être en lien avec des ressources ou avec un réseau naturel d'entraide dès son arrivée en ville. Des interventions permettant de répondre à ces besoins ont également été identifiées. Elles s'inscrivent dans une perspective de conscientisation et de responsabilisation collective sur la violence conjugale et misent sur l'autodétermination (empowerment) des femmes autochtones, de leur famille et de leur collectivité. 


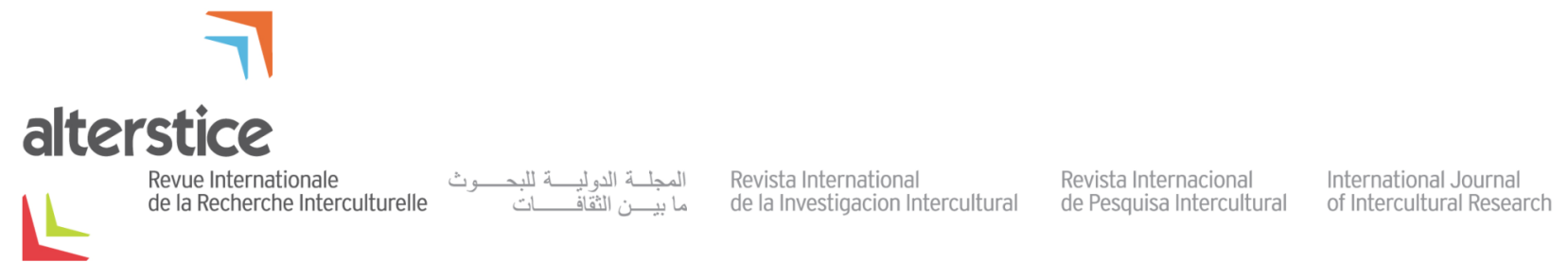

ARTICLE THÉMATIQUE

\title{
Sortir la violence de sa vie, sans sortir de l'autochtonie : l'importance de mieux comprendre les besoins des femmes autochtones en milieu urbain
}

\author{
Catherine Flynn ${ }^{1}$, Geneviève Lessard ${ }^{2}$, Lyse Montminy ${ }^{1}$ et Renée Brassard ${ }^{2}$
}

\section{Résumé}

Cet article présente les résultats d’une étude s'inscrivant dans une action concertée portant sur la violence conjugale envers les femmes autochtones du Québec. Notre recherche a porté sur les besoins et l'expérience de ces femmes en milieu urbain. Le corpus était constitué de trois groupes de discussion comprenant 24 hommes et femmes autochtones de Québec et de Montréal, tous concernés par la problématique de la violence conjugale. L'analyse a permis de dégager trois besoins en matière d'aide psychosociale : faire connaître la situation et le vécu des femmes autochtones victimes de violence conjugale, bénéficier d'une aide adaptée à la réalité culturelle des Autochtones et être en lien avec des ressources ou avec un réseau naturel d'entraide dès son arrivée en ville. Des interventions permettant de répondre à ces besoins ont également été identifiées. Elles s'inscrivent dans une perspective de conscientisation et de responsabilisation collective sur la violence conjugale et misent sur l'autodétermination (empowerment) des femmes autochtones, de leur famille et de leur collectivité.

\section{Rattachement des auteurs \\ ${ }^{1}$ École de service social, Université de Montréal, Montréal, Canada; ${ }^{2}$ École de service social, Université Laval, Québec, Canada.}

\section{Correspondance}

catherine.flynn@umontreal.ca

\section{Mots clés}

femmes autochtones; violence conjugale; services sociaux

\section{Pour citer cet article :}

Flynn, C., Lessard, G., Montminy, L. et Brassard, R. (2013). Sortir la violence de sa vie, sans sortir de l'autochtonie : l'importance de mieux comprendre les besoins des femmes autochtones en milieu urbain. Alterstice, 3(2), 27-xxx. 


\section{Introduction}

La violence conjugale envers les femmes autochtones est considérée comme une question sociale d'importance (Commission de la santé et des services sociaux des Premières nations du Québec et du Labrador [CSSSPNQL], 2003; Femmes autochtones du Québec inc. [FAQ], 2008). Des études populationnelles font état d'une prévalence variant entre $12 \%$ et $25 \%$, soit 3 à 4 fois plus élevée que dans la population allochtone (Brennan, 2011; Brownridge, 2003; Montminy et collab., 2011). Dans une population clinique, ces taux peuvent atteindre $80 \%$ voire 90 \% (Jones, 2008). Malgré le fait que les écrits scientifiques fassent état d'une situation alarmante, Hamby (2000) et Pharand (2008) rapportent que le nombre de femmes autochtones aux prises avec cette problématique demeure sous-estimé.

Il reste beaucoup à comprendre sur l'expérience des femmes autochtones victimes de violence conjugale. Des études comparatives montrent qu'elles sont plus susceptibles que les autres Canadiennes de subir des violences physiques ou sexuelles (Brownridge, 2003; FAQ, 2008; Tourigny, Domond, Trocmé, Sioui et Baril, 2007). La probabilité d'être victime d'un homicide conjugal à la suite d'une rupture serait huit fois plus élevée chez les femmes autochtones que chez les allochtones (Tourigny et collab., 2007). Elles sont également proportionnellement plus nombreuses à subir des blessures et à craindre pour leur vie lors d'un épisode de violence (Statistique Canada, 2006). Ces femmes courrent également un risque d'être financièrement exploitées et de subir de la violence mentale ou psychologique (Statistique Canada, 2001). La violence psychologique est d'ailleurs celle qui est la plus rapportée par les femmes autochtones qui amorcent une démarche auprès d'une ressource formelle d'aide (McGilivray et Comaskey, 1999).

La violence conjugale chez les Autochtones a de nombreuses conséquences néfastes chez les femmes et les enfants qui en sont victimes. Une étude de l'Agence de santé publique du Canada (ASPC, 2008) réalisée auprès d'intervenants et de femmes autochtones victimes de violence montre que ces dernières vivent des difficultés sur les plans physique, psychologique et social. Cette étude affirme que les femmes autochtones, tout comme les allochtones, ressentent de la honte et intériorisent les agressions vécues, croyant qu'elles sont méritées. Elle montre aussi que la violence entraîne une perte de l'estime de soi, ce qui représente un obstacle aux démarches de demande d'aide (Hines et Malley-Morrison, 2005).

Cet article examine l'expérience et les démarches de demande d'aide de femmes autochtones en milieu urbain et victimes de violence conjugale. Quels sont les principaux besoins en matière d'aide psychosociale des femmes autochtones en milieu urbain et victimes de violence conjugale ? Quelles sont les solutions à mettre en place pour faciliter leurs démarches? Dans un premier temps seront fournis quelques repères sociohistoriques abordés dans les écrits scientifiques, dont les facteurs associés à cette question en milieu autochtone. Ensuite seront abordés les enjeux soulevés dans la littérature relatifs aux démarches d'aide en milieu urbain. Nous présenterons en troisième lieu la méthodologie de notre recherche, puis ses résultats.

\section{Une violence construite et renforcée historiquement et socialement}

La problématique de la violence envers les femmes s'est construite de façon indissociable de l'histoire et des conditions de vie des peuples autochtones (ASPC, 2008; Hamby, 2000; Jones, 2008; Kasturirangan, Khrisnan et Riger, 2004). La Commission royale sur les peuples autochtones (CRPA, 1996) affirme que les Autochtones portent encore les séquelles de la colonisation et de la Loi sur les Indiens de 1876. Plusieurs auteurs soulignent que les politiques étatiques d'assimilation découlant de cette loi ont entraîné une discrimination systématique envers eux et une violence de l'État, qui est associée aussi à la problématique de la violence conjugale (ASPC, 2008; CRPA, 1996; Laprairie, 1987; Larocque, 1994; Pharand, 2008; Weaver, 2009). 
Ces auteurs expliquent que les transformations socioéconomiques issues de la Loi sur les Indiens ont perturbé les rôles traditionnels familiaux et les rapports égalitaires entre conjoints (Laprairie, 1987; Weaver, 2009). L'imposition du régime des écoles résidentielles de même que différentes mesures ciblant principalement les femmes ont contribué à dévaloriser leur rôle au sein des familles et des communautés (FAQ, 2008; Weaver, 2009). Pour certains, ces rapports de force entre l'État, les familles autochtones et les communautés ont laissé place à de la détresse et de la frustration, un terreau fertile à l'éclosion de la problématique de la violence conjugale et familiale (FAQ, 2008; Jaccoud et Brassard, 2003; Weaver, 2009).

Aujourd'hui, les femmes autochtones subissent encore les contrecoups des politiques assimilationnistes et du régime des écoles résidentielles. Elles souffrent notamment de traumatismes non réglés (Blackstock, Trocmé et Bennet, 2004) et de confusion identitaire (Brownridge, 2003) et elles ont une faible estime d'elles-mêmes ainsi qu'une perception négative de leur culture et de leur rôle social en tant que femme et mère (Smith, Varcoe et Edwards, 2005). Ces blessures trouvent d'ailleurs un écho dans la façon dont les femmes autochtones exercent leur maternité et elles teintent leurs rapports avec les services sociaux allochtones associés à la famille (Flynn et Brassard, 2012). À cela se greffent les réalités contemporaines des Autochtones, qu'on pense aux conditions de pauvreté et à l'exclusion sociale (Blackstock, Trocmé et Bennet, 2004; Jones, 2008), au suicide, à l'alcoolisme et à la toxicomanie (Smith, Varcoe et Edwards, 2005) ou à la surreprésentation des jeunes Autochtones dans les services de protection (Tourigny et collab., 2007). Pour plusieurs, la problématique de la violence conjugale est le produit d'une interaction entre l'héritage colonial et les différents facteurs de risque actuels (ASPC 2008; Brownridge, 2003; CSSSPNQL, 2003; FAQ, 2008; Jones, 2008; Pharand, 2008).

L'expérience des femmes victimes de violence conjugale dans les communautés autochtones s'avère marquée par la difficulté à dénoncer la situation et à s'en sortir. Les ressources d'hébergement étant limitées et l'anonymat difficilement garanti dans un milieu démographiquement restreint, plusieurs de ces femmes voit leur réalité révélée au grand jour. Elles sont alors sujettes à la marginalisation et aux pressions familiales, et elles craignent les représailles de leur conjoint et de sa famille. Dans un tel contexte, la fuite vers la ville leur apparaît souvent comme une solution afin de faire sortir la violence de leur vie (FAQ, 2008; Pharand, 2008).

\section{En milieu urbain : entre efforts et inadéquation}

La fuite afin d'échapper à une situation de violence conjugale est l'une des principales raisons expliquant la forte représentation des femmes autochtones dans la région de Montréal (Jaccoud et Brassard, 2003). Bien que peu d'études soient disponibles sur leur fréquentation des ressources d'aide en milieu urbain, La CSSSPNQL (2003) estime que ces femmes sous-utilisent ces services car ils ne répondraient pas à leurs besoins et leur imposeraient des valeurs allochtones. L'étude de Pharand (2008), réalisée dans la région de Montréal, montre d'ailleurs que les intervenantes des maisons d'hébergement allochtones connaissent peu la réalité des femmes autochtones et qu'elles croient, à tort, que leurs besoins sont similaires à ceux des autres femmes. L'association FAQ (2008) estime pour sa part que les ressources en milieu urbain sont peu visibles et peuvent se révéler difficiles d'accès. La FAQ se montre très claire sur sa position à propos du système d'aide allochtone spécialisé en violence conjugale, jugeant que ce dernier revictimise les femmes autochtones par sa complexité organisationnelle et est discriminatoire et raciste (FAQ, 2008).

On peut également supposer que des divergences de représentations (vision holistique autochtone face à la vision curative allochtone) sont susceptibles d'entraîner des difficultés pour les femmes autochtones dans leurs démarches de recherche d'aide en milieu urbain. Par exemple, les politiques publiques ont contribué à déprécier la culture, les croyances et le mode de vie des Autochtones, les forçant à se conformer à la vision du monde du colonisateur (Weaver, 2009). Les modes de guérison autochtones ont d'ailleurs été dissimulés et interdits jusque dans les années 1970 (Hill, 2003). Pour la FAQ (2008) et Weaver (2009), les réseaux d'aide allochtones interviennent auprès des femmes autochtones victimes de violence conjugale à partir d'une représentation de la problématique et de ses solutions qui est en inadéquation avec la représentation de la santé et du bien-être des Premières Nations. Les femmes autochtones des régions de Québec et de Montréal peuvent néanmoins accéder à une maison d'hébergement qui leur est destinée et qui utilise des formes d'aide adaptées à leur culture et à leur

Alterstice - Revue Internationale de la Recherche Interculturelle, vol. $3, n^{\circ} 2$ 
expérience. Il s'avère cependant que ces maisons ne suffisent pas à la demande (FAQ, 2008) et font face à différents obstacles sur le plan du financement (Pharand, 2008).

Malgré l'intérêt des chercheurs et des praticiens, les besoins des femmes autochtones victimes de violence conjugale demeurent un champ qui reste à explorer. Bien que quelques études (FAQ, 2008; Pharand, 2008), majoritairement menées par des organismes autochtones, se soient penchées sur cette question en laissant la parole aux femmes, il nous reste encore beaucoup à apprendre sur les liens entre l'expérience et les besoins de ces femmes, principalement en milieu urbain.

\section{Méthodologie de la recherche}

Cette étude est fondée sur une analyse des données secondaires extraites d'une action concertée sur les connaissances et des pratiques auprès des femmes autochtones victimes de violence menée par Montminy et ses collaboratrices (Flynn, 2010). Elle s'inscrit dans un paradigme interprétatif où subjectivité et processus inductif se côtoient. II s'agit d'effectuer une démarche exploratoire afin de dégager les principaux besoins en matière d'aide exprimés par les femmes autochtones et victimes de violence conjugale des régions de Québec et de Montréal. La méthodologie choisie est entièrement qualitative.

\section{Fondements théoriques}

Cette recherche s'inscrit également dans la foulée des théories critiques ayant le féminisme intersectionnel comme cadre d'analyse. Plus spécifiquement, nous avons mobilisé l'intersectionnalité et la matrice de la domination de Collins (2000). Cette matrice organise les différents systèmes d'oppression (patriarcat, colonialisme, etc.) selon quatre domaines de pouvoir, soit les domaines hégémonique, structurel, disciplinaire et interpersonnel. Le domaine hégémonique renvoie à l'idéologie, à la culture et aux messages sociaux véhiculés dans la collectivité. Les valeurs et les différents rapports d’oppression observés dans le domaine hégémonique influencent les manifestations du pouvoir dans les trois autres domaines. Le domaine structurel, pour sa part, illustre la façon dont les politiques et institutions publiques reproduisent l'oppression envers les femmes des groupes marginalisés. Le domaine du pouvoir disciplinaire réfère à la manière dont les rapports de pouvoir sont gérés dans les services publics comme le réseau de la santé, dans les entreprises, dans les banques, etc., et comment leurs pratiques contribuent à réguler la clientèle. Finalement, le domaine de pouvoir interpersonnel comprend les manifestations de pouvoir dans les interactions habituelles, de la vie quotidienne, dont la violence conjugale (Collins, 2000).

La réalisation d'une étude en conformité avec les principaux postulats du féminisme intersectionnel implique certains choix méthodologiques. Tout d'abord, cette théorie propose de privilégier le point de vue des groupes de femmes marginalisées et d'étudier leurs représentations de la violence dont elles sont victimes (Damant et collab., 2008). Dans le cadre de cette étude, la parole est laissée aux hommes et aux femmes qui estiment être concernés par la violence conjugale. Les critères d'inclusion ne sont pas déterminés par une représentation préétablie de la violence conjugale, ni par un rôle précis attribué è chacun et chacune dans cette dynamique. Laisser la parole aux hommes et aux femmes est cohérent avec la représentation autochtone de la violence conjugale, qui est plus souvent perçue comme un phénomène familial que comme relevant d'un rapport dyadique entre les deux conjoints (Montiny et collab., 2011). Le féminisme intersectionnel suggère également comme source d'information l'accès à des groupes de femmes marginalisées pour documenter leur réalité et leurs conditions de vie, afin de dégager les principaux traits de leur expérience individuelle et collective (Damant et collab., 2008). C'est la raison pour laquelle nous avons choisi comme stratégie de collecte de données la création de groupes de discussion.

\section{Participants}

Les participants devaient se considérer comme Amérindiens, Métis ou Inuit, être âgés d'au moins 18 ans et vivre à Québec ou à Montréal au moment de l'étude. Ils devaient également se sentir concernés par la problématique de la violence conjugale envers les femmes autochtones. Alors que plusieurs femmes ont choisi de parler de leur propre expérience de victimisation en lien avec la violence conjugale, d'autres ont choisi de partager celle d'une femme de leur entourage. Les hommes, pour leur part, ont principalement discuté des difficultés et des problèmes vécus par les hommes autochtones en milieu urbain. Ils ont également partagé leur expérience lors de 
l'accompagnement d'une femme de leur entourage au sein des différents services spécialisés en violence conjugale. L'échantillon était composé de 14 participants autochtones de la région de Québec (13 femmes et 1 homme), rencontrés en 2 groupes de discussion, et de 10 participants autochtones de Montréal ( 6 femmes et 4 hommes), réunis lors d'une séance commune. Afin que les participants puissent participer à cette étude de façon libre et anonyme, aucune information sociodémographique n'a été colligée. Les participants ont été recrutés par la méthode boule de neige (Pires, 1997) au sein des réseaux des principaux partenaires du projet, soit la Maison communautaire Missinak de Québec et le Foyer pour femmes autochtones de Montréal.

Nous avons ciblé les régions de Québec et de Montréal car le mouvement migratoire des Autochtones vers les villes du Québec se concentre principalement vers ces deux agglomérations (Lévesque, 2003). Les données ont été collectées par l'entremise des trois groupes de discussion (focus group), chacun d'une durée de deux heures. Cette méthode de collecte semblait appropriée car les Autochtones ont plus de facilité à exprimer leur vécu avec d'autres membres de leur communauté ou d'autres personnes d'appartenance autochtone (CSSSPNQL, 2003). Différents thèmes ont été abordés, en particulier les formes de violence vécues et observées par les participantes, les différentes stratégies pour s'en sortir, les obstacles rencontrés et les éléments ayant facilité leurs démarches de demande d'aide, de même que les interventions positives à mettre en place.

\section{Analyse qualitative}

Les données ont été traitées grâce à une analyse de contenu thématique selon la méthode proposée par L'Écuyer (1990). Cette méthode a semblé cohérente avec les principaux repères épistémologiques et théoriques de notre recherche puisqu'elle permet de systématiser l'analyse d'un matériel subjectif. Les différents thèmes des entrevues ont servi de catégories d'analyse. Le corpus a été codé une première fois dans ces catégories, puis les catégories redondantes ou contenant peu de matériel ont été réaménagées lors d'une seconde codification. Des analyses descriptives ont permis de dégager l'expérience des femmes en lien avec la violence conjugale et au sein des différents réseaux d'aide. Les besoins ont été définis comme des écarts entre une situation idéale et une situation réelle observée. Ils ont été identifiés en regroupant dans un tableau les extraits relatifs au vécu des femmes, aux limites des formes d'aide disponibles actuellement et aux formes d'aide souhaitées. Ces besoins ont ensuite été repositionnés dans les quatre domaines de la matrice de la domination de Collins (2002) afin de faire l'objet d'une analyse structurelle. L'ensemble de ces analyses a été réalisé avec le logiciel NVivo.

\section{Résultats}

Ces analyses ont permis de dégager trois besoins principaux en matière d'aide psychosociale : faire connaître la problématique et le vécu des femmes autochtones victimes de violence conjugale, bénéficier d'une aide adaptée à sa réalité sociale et culturelle et enfin être en lien avec des ressources ou avec un réseau naturel d'entraide dès son arrivée en milieu urbain. Il importe de mentionner qu'il est impossible de généraliser ces besoins à l'ensemble de la population autochtone des régions de Québec et de Montréal. Ces besoins sont en effet fortement liés à l'expérience des participants dans leur communauté d'appartenance, de même qu'à leurs rapports avec leur culture et leur identité. Il importe également de noter que l'ancrage dans les valeurs traditionnelles autochtones varie d'un individu à l'autre, de même qu'entre nations autochtones.

\section{Faire connaître la problématique et le vécu des femmes autochtones victimes de violence conjugale}

De l'analyse des propos des participants se dégage l'idée que la violence envers les femmes autochtones est banalisée. De ce fait, la façon d'y répondre tant dans les communautés qu'en milieu urbain crée un contexte susceptible de la faire perdurer. Cette banalisation, combinée à la méconnaissance par les intervenants que les participants ont côtoyés de la spécificité de cette problématique en contexte autochtone et des conditions sociohistoriques y étant associées montre qu'il est important de faire connaître le vécu des femmes qui sont victimes de violence conjugale. Ainsi, les participants estiment qu'il est nécessaire de réfléchir collectivement sur la violence conjugale chez les Autochtones et de sensibiliser l'ensemble des acteurs concernés par cette problématique, tant en milieu urbain que dans les communautés. 
« La violence $[. .$.$] c'est naturel»$

Les participants ont décrit la violence envers les femmes autochtones comme un mode relationnel banalisé et toléré dans leurs communautés. Lorsque des femmes de leur entourage ont vécu des épisodes de violence physique d'une extrême brutalité et en ont présenté les marques, personne n'a dénoncé la situation. Ils dénoncent le fait que la violence soit si présente qu'elle en devienne invisible.

C'est vraiment, dans les communautés, tu vois la violence, c'est tellement, tu vis dans ça. C'est naturel. Tu normalises la situation, fait que tu ne le vois pas. [...] C'est comme si c'était normal que la femme soit battue, c'est l'homme qui menait.

Dans ce climat, les participants estiment que les femmes qui en sont victimes sont poussées à se résigner. Les participants ont expliqué que les femmes subissent la pression des générations de femmes précédentes et de celles de leur entourage, le modèle de victimes silencieuses apparaissant particulièrement valorisé dans les communautés. Des femmes ont raconté avoir résisté à ces pressions et avoir pris la décision, marginale, de porter plainte.

Puis là, la famille me disait, pourquoi est-ce que tu fais ça ? Regarde, nous on ne fait pas ça, il nous trompe, il nous bat, on ne fait pas ça de porter plainte. Mais moi, on dirait que je n'arrivais pas à accepter ça.

Le soutien des proches et de la communauté étant difficile à obtenir, les participants croient que les femmes autochtones peuvent être tentées de se tourner vers les ressources du réseau formel d'aide qui sont disponibles dans leur communauté. Ils expliquent cependant que d'aller vers une maison d’hébergement n'est pas la meilleure solution.

Ça brise l'anonymat, des fois, dans nos communautés parce que tout le monde se connaît. Parce que les maisons d’hébergement sont situées dans le village, puis tout le monde le sait.

Celles qui choisissent de s'y rendre sont alors susceptibles d'être reconnues et d'être pointées du doigt. Dans un contexte où elles subissent des pressions sociales de leur famille et de leur entourage pour rester auprès d'un conjoint aux comportements violents et où elles sont stigmatisées par l'ensemble de la communauté en raison des difficultés liées à la confidentialité, les participants expliquent qu'il peut être préférable pour certaines femmes de quitter leur communauté, une étape difficile en soi.

Ce n'est pas évident de s'en sortir. [...] C'est fuir, tu sors de la communauté, tu n'as pas une cenne, tu t'en vas en milieu urbain, il faut que tu sois ferrée un peu ou que tu aies vraiment peur de te faire tuer.

"Si tu ne connais pas l'autre, comment tu veux adapter tes services?"

Après avoir pris la fuite et quitté la communauté, plusieurs femmes amorcent une démarche d'aide en milieu urbain. Les participants expriment cependant des réserves quant aux habiletés et aux connaissances des intervenants allochtones concernant l'aide destinée aux femmes autochtones victimes de violence. Ils estiment par ailleurs que les intervenants qu'ils ont côtoyés sont conscients de ces lacunes. Ces difficultés se sont soldées pour plusieurs à une discontinuité de l'aide offerte, articulée autour d'une multiplication de références et de changements d'intervenants.

Parce qu'ils se sentent... ils [les intervenants allochtones] n'ont pas les outils nécessaires pour mieux intervenir auprès des Autochtones [...] quand je voulais parler de ça avec les intervenants, ils n'étaient pas en mesure de répondre à mes questions. Si je vivais certaines émotions, eux autres ils étaient mal à l'aise. Donc, ça coupait tout.

Cette situation est susceptible de générer du découragement et un sentiment de rejet, pouvant même conduire à l'abandon des démarches d'aide. Les participants estiment que les intervenants allochtones consultés n'ont pas toujours bien compris leur vécu et que leur façon d'être était parfois incohérente avec les besoins. 
C'est comme si, parfois, dans certaines ressources allochtones, on sentait qu'on appliquait un cadre qui ne nous convenait pas. Ils ne respectent pas nos silences ou notre temps d'introspection, c'est différent là. Eux autres ils parlent, puis ils parlent, puis ils [mettent] toutes sortes de conditions.

Les participants suggèrent que la méconnaissance de la réalité des Autochtones laisse parfois place à une attitude de jugement de la part des intervenants rencontrés, ce qui peut être lourd de conséquences sur leurs démarches de demande d'aide.

Ils ne connaissent même pas ce qui se passe dans nos communautés. Puis quand tu parles de ta communauté, eux autres " c'est comme, ah oui, c'est comme ça que ça se passe ", là tu te rabaisses, là tu te refermes, puis non, tu n'as pas envie de parler. Puis, le jugement aussi, qui entre là-dedans.

Les discours des participants montrent que leur expérience et celle de leurs pairs au sein des différentes ressources d'aide en milieu urbain sont marquées par une méconnaissance de ce qu'ils vivent, de leur réalité et des principes de bases de l'intervention auprès d'eux. Cette attitude est parfois interprétée comme un manque d'empathie face à la détresse des femmes autochtones victimes de violence, comme en témoignent également leurs propos touchant leurs expériences avec le service de police.

"Les policiers, zéro intervention, zéro compassion »

Bien que les policiers puissent représenter une aide nécessaire en situation d'urgence, les participants expliquent qu'il y a réticence à les contacter dans les cas où la violence perdure ou se reproduit en milieu urbain. Certaines participantes auraient eu peur d'être jugées et de ne pas avoir le soutien nécessaire. D'autres estiment que l'aide des policiers a été insuffisante pour assurer leur sécurité et pour porter assistance aux femmes victimes de violence. Les participants expliquent que les interventions des policiers ont visé à contrôler la violence du conjoint plutôt qu'à assurer la protection de sa victime, et ce, même quand cette dernière présentait des blessures. Ils croient que le protocole d'intervention diffère lorsqu'il s'agit d'une situation impliquant des femmes allochtones et que les policiers demeurent insensibles en face de femmes autochtones victimes de violence. Les participants donnent d'ailleurs bon nombre d'exemples de situations où les policiers auraient dû porter assistance aux femmes et n'ont rien fait. Ils attribuent cette inaction aux préjugés et au racisme à l'égard des Autochtones.

II y avait une femme autochtone que je connaissais, qui était là avant moi, puis, elle pleurait, c'était très évident qu'elle s'était battue. Elle avait les yeux au beurre noir, on voyait où le monsieur avait donné des coups de poing, sur ses bras, ça se voyait très facilement. On a essayé de l'aider du mieux qu'on pouvait, même à la police, ils se sont fait appeler. [...] puis ils n'ont vraiment pas pris cette femme-là au sérieux, ils n'ont rien fait... [...] il faut dire que c'était des agents policiers blancs qui sont venus [...] [et] parce que c'était une femme autochtone, ils ne l'ont pas prise au sérieux.

Les participantes disent également avoir ressenti du jugement de la part des policiers lorsqu'elles ont porté plainte. Selon elles, plusieurs femmes craignent que leurs dépositions soient refusées ou invalidées.

La confiance des policiers, moi j'ai pas aimé le jugement des policiers. Parce que tu te dis, je vais faire une plainte, il ne prendra pas ma plainte, c'est moi qu'ils vont juger.

Le sentiment d'être jugée s'avère exacerbé lorsque la femme a recours aux policiers en état de consommation d'alcool ou de drogue, les participants faisant le lien entre la violence et la consommation de psychotropes. À ce moment-là, la méfiance de la femme s'ajoute à celle du policier qui reçoit le témoignage d'une personne intoxiquée. Les participants ont également eu des difficultés à obtenir l'aide d'un traducteur dans les échanges avec les agents, rendant ainsi le dépôt d'une plainte impossible.

Ainsi, l'analyse des propos des participants illustre qu'il est important de sensibiliser les intervenants et les policiers à la violence envers les femmes autochtones ainsi qu'aux circonstances sociales et historiques dans lesquelles cette violence a pris racine. Ces dernières peuvent d'ailleurs accentuer la méfiance envers les policiers et les intervenants allochtones. Un manque de connaissances ou de ressources peut être alors interprété comme une manifestation de racisme. Il est recommandé de mettre en place différentes interventions structurelles permettant d'outiller les 
intervenants, et les citoyens dans leur ensemble, à mieux comprendre cette réalité. La mise sur pied d'activités d'information, de prévention et de sensibilisation par le biais d'ateliers semble faire l'unanimité auprès des participants. Différentes thématiques en lien avec la violence pourraient y être abordées, comme les manifestations interpersonnelles et structurelles de la violence, l'histoire coloniale et les conditions de vie actuelles des Autochtones. Ces ateliers permettraient d'offrir du soutien au réseau d'aide local tant en milieu urbain que dans les communautés et contribueraient à déconstruire les stigmates et les préjugés envers les Autochtones. L'étude de Pharand (2008) montre d'ailleurs que les intervenants allochtones rencontrés sont conscients de leur manque de connaissances relatives aux réalités autochtones et qu'ils sont curieux d'en savoir davantage.

\section{Bénéficier d'une aide adaptée à sa réalité sociale et culturelle}

L'analyse des propos des participants a également permis de mettre en relief un deuxième besoin, celui de bénéficier d'une aide adaptée à sa réalité sociale et culturelle. Rappelons que les spécificités culturelles et sociohistoriques des Autochtones demeurent méconnues par les professionnels rencontrés par les participants. Les représentations de la violence conjugale et de ses solutions proposées par les ressources consultées se sont ainsi avérées en inadéquation avec les repères culturels des participants. Certaines approches et méthodes d'intervention privilégiées dans les milieux de pratiques allochtones s'opposent à leurs valeurs. Les participants ont proposé plusieurs solutions à privilégier afin d’obtenir, en milieu urbain, une aide adaptée à leur réalité.

"Ce n'est pas la façon de faire ça, ce n'est pas notre façon d'être "

Les participants se sont montrés très critiques envers les ressources allochtones consultées et ils ont expliqué en quoi elles n'ont pas respecté leurs différences et n'ont pas répondu à leurs besoins. Les participantes ayant fait l'expérience d'un séjour en maison d'hébergement allochtone expliquent avoir eu des difficultés avec les principes de l'intervention féministe. Elles ont compris que dans cette approche la rupture de l'union était un objectif. Selon elles, il s'agit d'une divergence de représentations concernant la famille.

Parce qu'il y a des enfants en jeu, puis dans la culture autochtone, élever des enfants toute seule, ce n'est pas faisable, ce n'est pas dans notre mentalité. Des femmes monoparentales, au Québec, il y en a beaucoup, mais en communauté, c'est rare.

Des participantes expliquent avoir ressenti la pression des intervenantes pour mettre un terme aux contacts avec le père de leurs enfants. Elles déplorent aussi que l'aide en violence conjugale se décline généralement sous la forme d'un suivi individuel. Chaque membre de la famille touchée par le problème reçoit de l'aide de son côté, et ce, généralement au sein de ressources différentes.

[Nous] on travaille avec la famille au lieu de travailler dans l'individuel, femme, homme. [Pour les Blancs] il faut toujours que ça soit un petit dossier pour madame, un petit dossier [...] on veut faire à la place de, puis imposer ses choses. Puis moi, je me dis toujours, on ne connaît pas notre histoire, on ne connaît même pas notre pensée, on n'a pas une pensée de Blanc là. [...] On n'a pas la même pensée pantoute, ce n'est pas la même façon dans notre tête.

Les participants ont décrit la problématique de la violence envers les femmes autochtones comme une dynamique complexe, soutenue et renforcée par la famille et la communauté. Par le biais de la pression qu'ils exercent sur les femmes, par leur inaction dans les cas où la violence est dénoncée ainsi que par certains comportements violents directement perpétrés, les membres de la famille élargie ou de la communauté sont directement impliqués. Ce " débordement » de la violence conjugale apparaît peu compris par les intervenants allochtones rencontrés par les participants. Ces derniers ont également la préoccupation de ne pas camper les acteurs impliqués dans des rôles dichotomiques de victimes et d'agresseurs. Il leur semble important de considérer la détresse et le vécu traumatique des conjoints qui perpètrent la violence. II s'agit de mettre la violence en lien avec l'ensemble de l'expérience des différents membres de la famille, et non de la traiter comme un problème isolé et décontextualisé. Ainsi, les participants croient que l'ensemble des membres de la famille devrait être impliqué conjointement dans une démarche d'aide globale. Cette stratégie a d'ailleurs été un succès pour certains participants de l'étude. 
La famille, tous ensemble, de venir avec nos problèmes sur la table, ça marche, crois-moi, ça marche, tout le monde évolue, mais d'une bonne manière. [...] Ça a pris des années d'entraide, entre nous autres, crois-moi, on s'est beaucoup amélioré. C'est très important l'aspect familial.

Les participants ont également rappelé l'importance de faire de la prévention auprès des enfants exposés à la violence. Ils se sont montrés soucieux de ce que ces derniers puissent reproduire ces comportements violents et qu'ils grandissent avec des traumatismes non résolus. Du point de vue des participants, l'aide destinée aux hommes autochtones apparaît également incontournable, considérant qu'il n'y a pas de ressource de ce type dans les régions de Montréal et de Québec. Les participants recommandent d'ailleurs l'implantation d'un programme comme celui des Peaceful Warriors, un service destiné aux hommes axés sur l'enseignement traditionnel autochtone implanté au Native child and family services de Toronto. La sagesse des aînés peut également être mise à profit, ceux-ci étant considérés comme des gens ayant une vaste expérience et ayant été impliqués dans des processus de guérison.

\section{"Avec du monde autochtone, ça se passait mieux »}

Il est ressorti des analyses que l'aide formelle dispensée par des intervenants ou des ressources autochtones a été appréciée par les participantes. II semblerait que les intervenants autochtones comprennent mieux leur vécu ainsi que leurs spécificités culturelles et spirituelles, même si leur aide n'est pas spécifiquement destinée à contrer la violence conjugale.

On comprend les choses d'une manière différente, d'une autre façon que les Blancs, c'est géré d'une autre manière. Eh oui, je pense que je suis mieux là-dedans, avec des intervenants autochtones, parce qu'ils sont autochtones et parce que c'est mon monde à moi et parce que, notre culture, on comprend ça d'une manière complètement différente.

Quelques participants avancent la nuance que certains de ces intervenants puissent être encore aux prises avec leurs propres blessures en lien avec l'expérience des écoles résidentielles, ces traumatismes non résolus interférant dans la démarche d'aide. Néanmoins, les participants croient que l'appartenance à l'autochtonie est une base commune sur laquelle il est plus facile de créer une relation d'aide. Ainsi, ils estiment que les femmes autochtones victimes de violence conjugale s'expriment plus facilement avec des intervenants autochtones. Côtoyer l'autochtonie leur apparaît comme un élément facilitant les démarches d'aide. Les modes d'intervention efficace proposés par les participants s'inscrivent dans cette perspective.

\section{"On veut quand même que nos services soient autochtones, on ne se le cachera pas »}

Les solutions proposées par les participants visent une autonomisation de l'aide et des services offerts en milieu urbain. Il est possible d'y voir un désir de s'affranchir des ressources du réseau allochtone et de développer des formes d'aide en adéquation avec leur culture. Il a été suggéré de poursuivre l'implantation de formes d'aide traditionnelle autochtone, comme des cercles de partage et des tentes de sudation, qui soient offertes à une clientèle féminine et masculine.

Entre nous, tu sais, d'avoir des « healing circle » ou avoir des « swet lodge " contre la violence, des prières contre la violence puis des affaires comme ça.

Il a été également recommandé de créer des espaces afin que les protagonistes impliqués dans une dynamique de violence puissent prendre un temps d'introspection sur leur propre expérience en lien avec la violence. Les participants ont mentionné les difficultés à recevoir de l'aide en langue autochtone et ont insisté sur les difficultés à nommer ses émotions dans une autre langue. D’autres ont expliqué que la culture autochtone prend racine dans les langues ancestrales, et qu'il est donc primordial de pouvoir s'y ancrer dans une démarche d'aide.

La langue, c'est essentiel, parce qu'il y a des personnes qui ne peuvent pas s'exprimer. Moi j'ai eu de la misère à dire mes émotions, c'est dur. [...] Ça (la langue), tu ne te débarrasses pas de ça, ça ne part pas de toi parce que tu habites en ville, ça reste en dedans de toi, c'est toi. 
Les participants de cette étude ont mis de l'avant l'autochtonie comme élément favorisant leurs démarches d'aide en milieu urbain. Ils ont relevé diverses inadéquations entre leurs représentations de la violence conjugale et les interventions proposées par les réseaux d'aide allochtones en milieu urbain. Néanmoins, quelques participants ont décrit des situations où l'aide obtenue de la part d'un intervenant allochtone a été appréciée. Un participant a ainsi expliqué que, malgré le manque de connaissances de l'intervenante sur la réalité des peuples autochtones, son attitude respectueuse, son écoute et son ouverture ont fait le succès de sa démarche.

\section{Être en lien avec des ressources ou avec un réseau naturel d'entraide dès son arrivée en milieu urbain}

Finalement, les participants ont cité l'isolement et à la difficulté à s'orienter dans un nouveau milieu de vie comme des éléments rendant plus complexe l'amorce d'une démarche d'aide en milieu urbain. Ils croient que les ressources d'aide aux Autochtones devraient être plus visibles et mieux connues dans les communautés. Ceci permettrait aux femmes d'être préparées lors de leur arrivée, cette transition s'étant effectuée dans un contexte de précarité pour plusieurs d'entre elles. Ces dernières soulignent qu'elles auraient eu besoin dès le début d'être en lien avec des ressources formelles ou un réseau naturel d'entraide.

\section{"Il faut se débattre, il faut vraiment tout faire pour aller chercher l'aide qu'il faut »}

Les participants ont estimé que les ressources spécialisées pour les Autochtones étaient peu visibles lors de leur arrivée en milieu urbain. Ils jugent que les stratégies pour les faire connaître (comme les affiches) sont inefficaces et qu'il serait préférable d'adopter d'autres modes de diffusion. Ils croient que l'on devrait fonctionner en amont, avec la clientèle potentielle, afin de rapidement briser l'isoler des femmes fuyant des dynamiques de violence. Par exemple, ils proposent la présence d'intervenants dans les lieux et les quartiers fréquentés par les Autochtones nouvellement arrivés. Des prospectus pourraient être distribués dans les endroits favoris de cette clientèle.

Qu'ils viennent se présenter, qu'ils offrent leurs services. [...] Si la personne vient et qu'elle parle des services, comment ça se fait, ce qu'ils font là-bas, peut-être que les femmes vont se réveiller. Elles viennent de sortir de leur communauté, ah, j'aimerais ça avoir de l'aide. .]. Plus de promotion des services qu'on connaît moins.

Les participants ont aussi proposé de créer un groupe de lutte contre la violence envers les femmes autochtones. Cette initiative permettrait de dynamiser l'aide aux femmes et ferait office de guichet unique et de point d'ancrage pour les démarches subséquentes de demande d'aide. Ce groupe permettrait également de réfléchir collectivement sur cette problématique et en faire une responsabilité collective.

"Si quelqu'un te tend la main [...] ça aide !»

Il est également suggéré de favoriser les occasions de réseautage entre femmes autochtones afin de briser cet isolement et de créer un réseau naturel d'entraide.

Chez tous les Autochtones, quand ils arrivent, c'est de se trouver un point de repère. [...] juste aller prendre un café, pas nécessairement faire quelque chose d'officiel [...] On invite du monde, un souper, juste ça [...] ça les sort de leur isolement.

Il importe de mentionner que les participantes ont apprécié le soutien de leur nouveau réseau informel en milieu urbain. La préférence pour un réseau naturel d'entraide plutôt que pour des ressources formelles est apparue très forte auprès des participants en provenance de Québec. Ceux-ci ont expliqué que les femmes autochtones de leur région comptent les unes sur les autres pour se dépanner ou se cacher lorsque c'est nécessaire. Ils ont aussi expliqué que, contrairement à ce qui se passe dans leur communauté, un réseau informel de protection et de soutien se crée autour de la femme victime de violence.

Puis, il y avait une fille qui était violentée par son conjoint, puis c'est ça qu'on faisait, on l'aidait. Des fois, elle venait dormir chez nous, [...] elle pouvait aller dormir chez une autre collègue étudiante, c'est ça qu'elle faisait, elle voulait se cacher aussi.

De tels réseaux d'entraide se sont créés entre des femmes qui se sont connues par le biais d'un centre de formation fréquenté par les Autochtones ou par les activités organisées par une maison d’hébergement 
autochtone de la région de Québec. Le contact avec d'autres femmes autochtones en milieu urbain ainsi que la participation à des activités informelles en lien avec la culture se sont révélés bénéfiques pour les participants. Ces échanges leur ont permis de développer un sentiment d'appartenance à cette nouvelle " communauté » et de renforcer leur solidarité. Les participants de Québec ont d'ailleurs mentionné que l'aide reçue par leurs pairs en milieu urbain et le discours exempt de jugement de ces derniers contrastent avec le contexte victimisant et culpabilisant de leur communauté d'origine. Cette réalité de la région de Québec tranche avec les propos des participants de la région de Montréal, où les femmes autochtones semblent plus éparses et où la mouvance entre Montréal et les communautés (une forme moderne de nomadisme) représente un obstacle à leurs efforts de solidarisation.

Je pense qu'on a plus de problèmes comme communauté, ici à Montréal, parce qu'il y a trop de down andin [...] on ne parle pas assez de ce qui se passe, puis on n'a vraiment pas de recours pour s'exprimer. On devrait être capable, pour moi, de se mettre en cercle, puis exprimer n'importe quoi qui se passe dans notre tête, dans notre vie personnelle.

Le réseautage entre les femmes autochtones en milieu urbain et celles en communautés a également été évoqué comme solution pour les nouvelles arrivantes et pour celles qui projettent de quitter la communauté. Encore une fois, ce besoin exprimé montre qu'il a été bénéfique pour les participants de côtoyer l'autochtonie en milieu urbain. Il s'avère important d'offrir également soutien et aide à s'orienter au sein du réseau formel d'aide en milieu urbain.

\section{Discussion : banalisation, méfiance et ambivalence}

Cette recherche a permis de mettre en relief la complexité et l'étendue de la problématique de la violence conjugale envers les femmes autochtones en milieu urbain. L'étude exploratoire a permis de mieux cerner les besoins de ces femmes. Nous avons analysé les discours de 24 participants autochtones, hommes et femmes, touchant divers thèmes en lien avec cette problématique. Cette analyse a été réalisée à partir d'une grille d'interprétation féministe intersectionnelle, la matrice de la domination de Collins (2000). Nous avons positionné les différents obstacles aux démarches d'aide de même que les éléments relatifs aux pratiques d'aide dans les quatre domaines du pouvoir de cette matrice, les pouvoirs hégémonique, structurel, disciplinaire et interpersonnel.

Les expériences de ces femmes, telles qu'elles ont été dépeintes par les participants à nos groupes de discussion, sont toutes marquées par la difficulté à sortir la violence de leur vie en raison de nombreux obstacles systémiques. La tolérance à la violence est apparue comme un thème récurrent, la violence étant banalisée à différents niveaux. Dans le domaine hégémonique, la violence envers les femmes semble être devenue un phénomène naturel au sein des communautés, du fait de sa répétitivité et de son invisibilité. Les politiques assimilationnistes et discriminatoires envers les femmes autochtones ont également contribué à déprécier les rôles féminins et sont encore aujourd'hui porteuses de conséquences pour celles-ci. Les participants ont également souligné que les contingences socioéconomiques des communautés exacerbent la dépendance des femmes envers leur conjoint, ce qui représente un facteur de maintien dans la relation violente et contribue à exacerber la tolérance à la violence. Ces aspects importants, qui relèvent du domaine structurel, soutiennent les dynamiques de violence envers les femmes. Dans le domaine disciplinaire, les participants ont fourni de nombreux exemples où des femmes autochtones victimes de violence conjugale se sont senties peu considérées, ignorées ou dévalorisées. Les différentes réactions des policiers apparaissent symptomatiques de cette réalité. Finalement, dans le domaine interpersonnel, et en accord avec ce qui a été relevé dans divers écrits scientifiques (FAQ, 2008; Pharand, 2008), les femmes autochtones victimes de violence sont susceptibles d'être marginalisées par leur communauté et de subir des pressions familiales lorsqu'elles dénoncent la violence qu'elles vivent. Tous ces éléments tendent à complexifier les démarches à entreprendre pour mettre fin à la violence et au renforcement par les femmes et les hommes des communautés de la tolérance envers cette violence.

Le repositionnement à l'intérieur de la matrice de Collins (2000) des expériences et des besoins dont les participants ont témoigné, principalement dans les domaines hégémoniques, structurels et disciplinaires, illustre aussi le fait que la relation entretenue par les femmes autochtones victimes de violence vis-à-vis des ressources allochtones puisse être marquée par la méfiance. Cette méfiance envers les allochtones s'est construite 
historiquement, entretenue par les politiques colonialistes, et elle s'actualise dans l'interprétation que les participants font des rapports avec les intervenants des ressources formelles. La méconnaissance par ces derniers de l'histoire coloniale et des conditions de vie actuelles des peuples autochtones, tout comme les lacunes dont ils font preuve lorsqu'ils communiquent et interviennent auprès des Autochtones, peuvent alors être rapidement perçues comme les manifestations d'un racisme ou d'une discrimination. De même, un modèle d'intervention en inadéquation avec les représentations que les participants ont de la violence conjugale peut alors être interprété comme l'imposition d'un mode de pensée allochtone et une reproduction du pouvoir allochtone. Les séquelles des politiques assimilationnistes et ces interactions difficiles lors des contacts avec les différents services se combinent donc pour renforcer cette méfiance et raviver, dans les représentations des participants, les tensions entre Autochtones et allochtones.

Tous ces éléments ont comme conséquence de freiner les démarches de demande d'aide et de renforcer la préférence pour l'aide dispensée par des Autochtones et à partir de formes d'aide traditionnelles. Le fait que l'aide formelle et informelle autochtone soit préférée à l'aide allochtone est cependant le signe d'une certaine ambivalence. D'un côté, on trouve la volonté de briser le lien avec la communauté pour sortir de la violence. De l'autre émerge le désir de la femme d'être aidée par un réseau formel ou informel en conformité avec ses valeurs. Cette contradiction témoigne de la complexité du vécu des femmes, et montre que l'analyse de leur situation et la recherche de solutions impliquent la prise en compte d'une dimension systémique et de différents éléments culturels et historiques. Cette ambivalence des femmes face à leur ancrage identitaire et culturel demeure une piste de recherche à approfondir.

Plus généralement, il apparaît difficile d'appréhender la violence conjugale envers les femmes autochtones à l'extérieur de sa dimension culturelle, étant donné que les différentes nations autochtones conçoivent les dimensions spirituelle, psychologique, physique et émotionnelle comme un tout cohérent dans la production de la santé et du bien-être, ce qui est loin d'être le cas dans le reste de la société (CSSSPNQL, 2003; FAQ, 2008; Hill, 2003; RCRPA, 1996). Selon les participants aux groupes de discussion, les femmes souhaitent recevoir une aide qui favorise les initiatives autochtones, permette la revalorisation de leur culture, respecte leurs différences culturelles et soutienne l'autodétermination (empowerment) pour elles-mêmes, leur famille et leur collectivité. Des chercheures et des intervenantes allochtones tentent déjà de repenser l'intervention féministe afin d'y intégrer certains éléments relatifs aux enjeux interculturels, afin de mieux répondre aux besoins des femmes issues de la diversité (Marchand et Ricci, 2011).

Les témoignages des participants et la réflexion en cours sur l'intervention féministe sont le signe d'une ouverture à agir au-delà du cadre autochtone. Les besoins exprimés et les solutions proposées par les participants offrent la preuve que la violence conjugale envers les femmes autochtones concerne un vaste éventail d'acteurs, et les participants ont formulé explicitement le souhait que cette lutte contre la violence intègre un niveau de responsabilité collective, qu'elle soit partagée par tous. Briser le cycle de la méfiance nécessite par exemple une curiosité de la part des intervenants à l'égard du vécu et des conditions de vie des peuples autochtones, de même qu'une attitude d'acceptation et de non-jugement. Plus globalement, cela implique d'explorer des modes d'interventions qui sollicitent la participation des différents acteurs concernés par la problématique et d'instaurer des rapports égalitaires entre tous.

\section{Références bibliographiques}

Agence de la santé publique du Canada (2008). Femmes autochtones et violence familiale. Ottawa : Agence de la santé publique du Canada, Centre national d'information sur la violence dans la famille.

Blackstock, C., Trocmé, N. et Bennet, M. (2004). Child maltreatment investigations amoung Aborignal and NonAboriginal families in Canada. Violence against women, 10, 901-916.

Brennan, S. (2011). La victimisation avec violence chez les femmes autochtones dans les provinces canadiennes, 2009 (rapport Juristat). Ottawa : Statistique Canada. ( $\left.n^{\circ} 85-002-X\right)$

Brownridge, D. (2003). Male partner against Aboriginal women in Canada: an empirical analysis. Journal of interpersonnal violence, $18,65-83$.

Alterstice - Revue Internationale de la Recherche Interculturelle, vol. 3, $n^{\circ} 2$ 
Collins, P. (2000). Black feminist thought: knowledge, consciousness and the politics of empowerment ( 2 éd. revue). New York et Londres : Routledge.

Commission de la santé et des services sociaux des Premières nations du Québec et du Labrador (CSSSPNQL). (2003). Adapter nos interventions à la réalité autochtone. Ottawa : Santé Canada.

Commission royale sur les Peuples autochtones (1996). Rapport de la Commission royale sur les Peuples autochtones. Perspectives et réalités (vol. 4). Ottawa : Ministère des approvisionnements et Services Canada.

Damant, D., Lapierre, S., Kouraga, A., Fortin, A., Hamelin-Brabant, L., Lavergne, C. et Lessard, G. (2008). Taking child abuse and mothering into account: Intersectional feminism as an alternative for the study of domestic violence. Affilia, 23, 123-185.

Femmes autochtones du Québec inc. (FAQ) (2008). Les femmes autochtones et la violence. Kahnawake, Québec : Femmes autochtones du Québec.

Flynn, C. (2010). Femmes autochtones en milieu urbain et violence conjugale. Étude exploratoire sur l'expérience et les besoins en matière d'aide psychosociale selon des hommes et des femmes autochtones concernés par cette problématique (mémoire de maîtrise, Université Laval, Québec). www.theses.ulaval.ca/2010/27765/27765.pdf

Flynn, C. et Brassard, R. (2012). La maternité autochtone en contexte de violence structurelle : entre repères culturels, négociations et hégémonie. Dans S. Lapierre et D. Damant (dir.), Regards critiques sur la maternité dans divers contextes sociaux (p. 102-124). Québec: Presses de l'Université du Québec.

Hamby, S. (2000). The importance of community in a feminist analysis of domesticviolence among American Indians. American journal of community psychology, 28, 649-669.

Hill, D. (2003). La guérison traditionnelle dans les contextes comptemporains : protéger et respecter le savoir et la guérison indigènes. Ottawa : Organisation nationale de la santé des Autochtones.

Hines, D. et Malley-Morrison, K. (2005). Family violence in the United States: defining, understanding, and combating abuse. Thousand Oaks, CA : Sage Publications.

Jaccoud, M. et Brassard, R. (2003). La marginalisation des femmes autochtones à Montréal. Dans D. Newhouse et E. Peters, Des gens d'ici - Les Autochtones en milieu urbain, Projet de recherche sur les politiques (p. 143160). Ottawa : Gouvernement du Canada.

Jones, L. (2008). The distinctive characteristics and needs of domestic violence victims in a Native American community. Journal of Family Violence, 23, 113-118.

Kasturirangan, A., Krishnan, S. et Riger, S. (2004). The Impact of Culture and Minority Status on Women's Experience of Domestic Violence. Trauma, violence and abuse, 5, 318-332.

Laprairie, C. (1987). Native women and crime: a theoretical model. The Canadian journal of native studies, 7, 121137.

Laroque, E. (1994). Violence in Aboriginal communities. Ottawa : Centre national d'information sur la violence dans la famille.

L'Écuyer, R. (1990). Méthodologie de l'analyse développementale de contenu. Québec: Presses de l'Université du Québec.

Lévesque, C. (2003). La présence des Autochtones dans les villes du Québec: mouvements pluriels, enjeux diversifiés. Dans D. Newhouse et E. Peters, Des gens d'ici-Les Autochtones en milieu urbain, Projet de recherche sur les politiques (p. 25-39). Ottawa: Gouvernement du Canada.

Marchand, I. et Ricci, S. (2010). Sexisme et racisme : la diversité ethnoculturelle, défi au mouvement féministe. Dans C. Corbeil et I. Marchand (dir.), L'intervention féministe d'hier à aujourd'hui. Portrait d'une pratique sociale diversifiée (p. 65-92). Montréal : Éditions du remue-ménage. 
McGilivray, A. et Comaskey, B. (1999). Black eyes all of the time: intimate violence, Aboriginal Women, and the justice system. Toronto : University of Toronto Press.

Montminy, L., Brassard, R., Jaccoud, M., Harper, É., Bousque, M.-P. et Leroux, S. (2011). Pour une meilleure compréhension des particularités de la violence familiale vécue par les femmes autochtones au Canada. Nouvelles pratiques sociales, 23, 53-66.

Pharand, S. (2008). Ishkuteu : Des services d'aides en violence conjugale en réponse aux besoins des femmes autochtones. Montréal : Fédération de ressources d’hébergement pour femmes violentées et en difficulté du Québec et Femmes autochtones du Québec.

Pires, A. (1997). Échantillonnage et recherche qualitative : essai théorique et méthodologique. Dans J. Poupart, J.-P. Deslauriers, L. Groulx, A. Laperrière, R. Mayer et A. Pires (dir.), La recherche qualitative : enjeux épistémologiques et méthodologiques (p. 113-167). Boucherville : Gaëtan Morin.

Smith, D., Varcoe, C. et Edwards, N. (2005). Turning around the intergenerational impact of residential school on Aboriginal people: implications for health policy and practice. Canadian journal of nursing research, 37(4), 39-60.

Tourigny, M., Domond, P., Trocmé, N., Sioui, B. et Baril, K. (2007). Les mauvais traitements envers les enfants autochtones signalés à la protection de la jeunesse du Québec : comparaison interculturelle. First People child and family rewiew, 3, 84-102.

Statistique Canada (2001). La violence familiale au Canada. Ottawa : Statistique Canada, Centre canadien de la statistique juridique.

Statistique Canada (2006). Mesure de la violence faite aux femmes - tendances statistiques. Ottawa : Statistique Canada.

Weaver, H. (2009). The colonial context of violence: Reflections on violence in the lives of American Indian women. Journal of interpersonal violence, 24, 1552-1563. 